\title{
Supercritical carbon dioxide fluid extraction of Hibiscus cannabinus L. seed oil: A potential solvent-free and high antioxidative edible oil
}

\begin{abstract}
The supercritical fluid extraction (SFE) trends and antioxidant activities of Hibiscus cannabinus seed oils were studied. SFE results indicate that extraction pressure is the major factor determining the oil yield. In comparison, classic Soxhlet extraction (SOX/L) yielded higher oil content than SFE (P \&lt; 0.05). However, no significant differences in oil content were observed in SFE at 600 bars $/ 80{ }^{\circ} \mathrm{C}$, rapid Soxhlet extraction $(\mathrm{SOX} / \mathrm{S})$ and conventional ultra-sonic assisted solvent extraction (SONIC) $(\mathrm{P}>0.05)$. Antioxidant activities of $\mathrm{H}$. cannabinus seed oils were compared with 7 types of commercial edible oils. DPPH scavenging activity test indicated that $\mathrm{H}$. cannabinus seed oil extracted by SFE at $200 \mathrm{bars} / 80$ ${ }^{\circ} \mathrm{C}$ possessed the highest antiradical activity whereas beta-carotene bleaching (BCB) assay revealed that all $\mathrm{H}$. cannabinus seed oils (except for SFE at $400 \mathrm{bars} / 80{ }^{\circ} \mathrm{C}$ and $600 \mathrm{bars} / 80$ ${ }^{\circ} \mathrm{C}$ ) exhibited higher antioxidant activity than all commercial edible oils (P \&lt; 0.05). Thus, SFE - H. cannabinus seed oil may serve as an excellent source of solvent-free edible oil with high antioxidant properties.
\end{abstract}

Keyword: Antioxidant activity; H. cannabinus seed oil; Supercritical carbon dioxide extraction. 